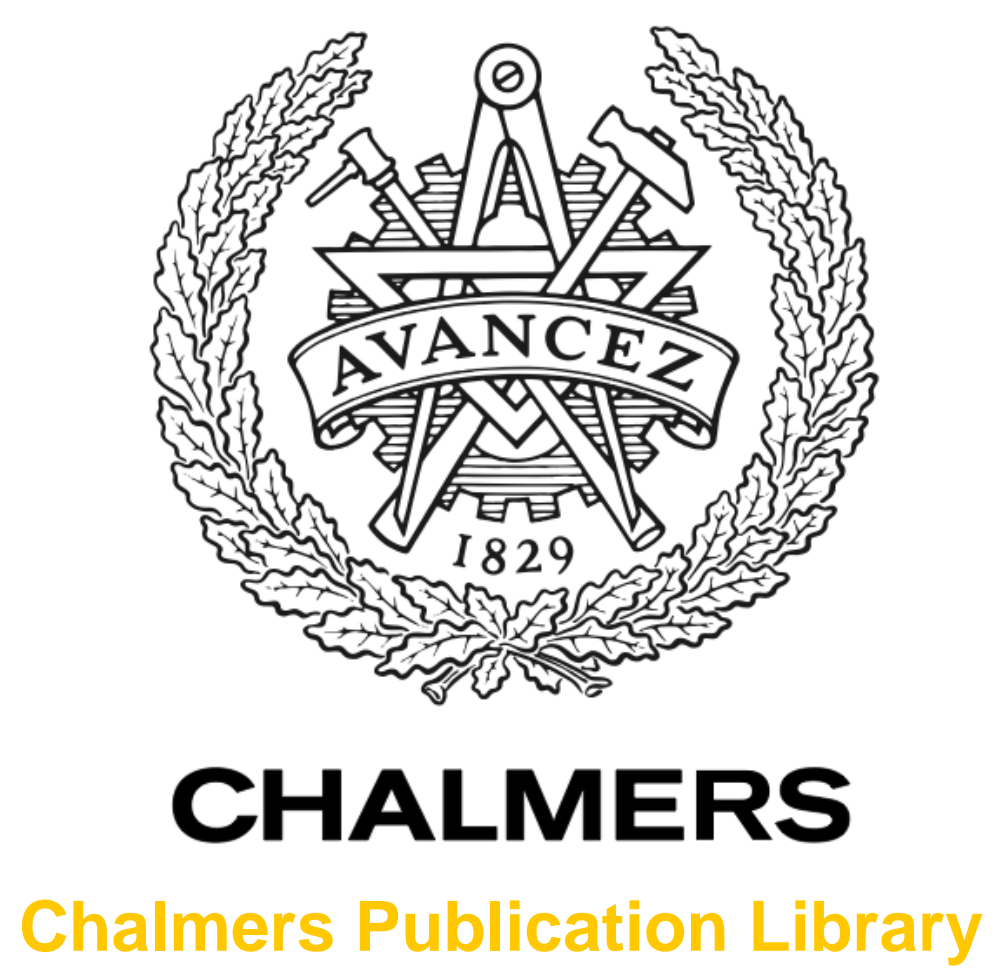

An integrated superconducting phase switch for cosmology instruments

This document has been downloaded from Chalmers Publication Library (CPL). It is the author's version of a work that was accepted for publication in:

Physica C-Superconductivity and Its Applications (ISSN: 0921-4534)

Citation for the published paper:

Yassin, G. ; Kuzmin, L. ; Grimes, P. (2007) "An integrated superconducting phase switch for cosmology instruments". Physica C-Superconductivity and Its Applications, vol. 466(1-2), pp. 115-123.

http://dx.doi.org/10.1016/j.physc.2007.06.014

Downloaded from: http://publications.lib.chalmers.se/publication/82323

Notice: Changes introduced as a result of publishing processes such as copy-editing and formatting may not be reflected in this document. For a definitive version of this work, please refer to the published source. Please note that access to the published version might require a subscription.

Chalmers Publication Library (CPL) offers the possibility of retrieving research publications produced at Chalmers University of Technology. It covers all types of publications: articles, dissertations, licentiate theses, masters theses, conference papers, reports etc. Since 2006 it is the official tool for Chalmers official publication statistics. To ensure that Chalmers research results are disseminated as widely as possible, an Open Access Policy has been adopted.

The CPL service is administrated and maintained by Chalmers Library. 


\title{
An integrated superconducting phase switch for cosmology instruments
}

\author{
Ghassan Yassin ${ }^{\text {a,*, Leonid S. Kuzmin }}$ b, Paul K. Grimes a, Mikhail Tarasov b, \\ Ernst Otto $^{\mathrm{b}}$, Phillip D. Mauskopf ${ }^{\mathrm{c}}$ \\ ${ }^{a}$ University of Oxford, Astrophysics, Denys Wilkinson Building, Keble Road, Oxford, OX1 3RH, UK \\ ${ }^{\mathrm{b}}$ Chalmers Tekniska Högskola, Gôteborg S-41296, Sweden \\ ${ }^{\mathrm{c}}$ Cardiff University, School of Physics and Astronomy, 5 The Parade, Cardiff, CF24 3 YB, UK
}

Received 25 December 2006; received in revised form 29 May 2007; accepted 11 June 2007

Available online 18 July 2007

\begin{abstract}
We present the design of a low loss planar phase switch operating at millimetre and submillimetre wavelengths. The system comprises a superconducting nanostrip that switches the RF signal between the two branches of a microstrip phase circuit. The employment of superconducting components reduces conduction losses to a negligible level. The required cooling below the transition temperature does not add extra effort since all high performance detectors employ superconducting circuits, and are in fact cooled well below the transition temperature of the phase circuit material. The proposed fully planar design allows the switch to be easily integrated into the detector circuit and eventually the realisation of a fully planar receiver. This avoids the use of bulky, expensive and lossy waveguide components and allows the fabrication of reliable and cheaply mass producible polarimeters that are now routinely used in large format CMB arrays. (C) 2007 Elsevier B.V. All rights reserved.
\end{abstract}

Keywords: Phase modulation; Superconducting nanostrip

\section{Introduction}

Polarisation of the cosmic microwave background (CMB) is caused by the Thompson scattering of photons by electrons near the last scattering surface during recombination. The existence of temperature anisotropy allows a small yet significant linear polarisation to be imparted on the scattered CMB photons [1]. The polarisation signal can be decomposed into a curl (B-mode) and a curl-free component (E-mode), each representing a particular distribution of the electromagnetic signals on the sky. The Emode component is generated by density perturbations, has an amplitude of approximately $10 \%$ of the temperature anisotropy and has recently been detected [2-4]. The Bmode however is generated entirely by primordial gravitational waves and provides unique information about the

\footnotetext{
* Corresponding author.

E-mail address: ghassan@astro.ox.ac.uk (G. Yassin).
}

early universe and would greatly increase our ability to constrain inflationary models.

Theoretical models however predict the amplitude of the B-mode signal to be at best an order of magnitude smaller than the E-mode and hence its detection constitutes a major technological challenge.

At present there are several ground based and balloonborne (Clover [5], EBEX [6], BICEP [7], QUIET [8]) cosmology instruments that are being designed or built to make the first detection of the B-mode component. The frequency of operation of these instruments ranges from 40 $450 \mathrm{GHz}$ and although they employ substantially different technologies they have an important feature in common: they all employ focal plane imaging arrays with approximately 100-200 pixels in each array. For example, the UK-led instrument Clover comprises three independent telescopes operating at 97,150 and $225 \mathrm{GHz}$ with $30 \%$ bandwidth and a beamwidth of approximately 8 arcminutes, covering the angular range of $20<l<1000$. The choice of this relatively large number of pixels is dictated 
by the exceptional sensitivity required to detect the B-mode component and yet is restricted by the technological difficulty of packing a large number of detectors at the focal plane of a compact telescope and also the huge expense of high precision machined components (hybrids, orthomode transducers, horns, etc.) at millimetre wavelengths.

The state of polarization of an electromagnetic wave can be uniquely determined by the measurement of the Stokes parameters $I, Q, U, V$ where $I$ is the total intensity, $V$ measures the degree of circular polarization and $Q$ and $U$ measure the degree of linear polarization in two orthogonal directions. This can be done using the pseudo-correlation polarimeter described in Fig. 1. Here the signal received by one of the focal plane horns is split into two linear polarizations using an OMT. The two signals are then converted into circular polarization using a quadrature hybrid and one of them is phase modulated with the respect to the other before they are converted back into linear polarization and fed to the detectors D1 and D2. Using the Jones matrices method, it can be shown that the outputs measured at the detectors are:

$D_{1}=I-Q \cos \psi-U \sin \psi$,

$D_{2}=I+Q \cos \psi+U \sin \psi$.

The above equation tells us that our output is sensitive to $I, U$ and $Q$ stokes parameters and that it is possible to determine the linear polarisation parameters simultaneously for a single sky pixel by taking the difference of the detector outputs, without moving the optics. For example, by switching the phase difference between 0 and $180^{\circ}$, the $U$ term remains zero and $Q$ is measured and similarly, by chopping the phase difference between 0 and $90^{\circ}$ we recycle the outputs between $Q$ and $U$.

There are two important advantages in employing phase modulation in conjunction with a correlation polarimeter. First it allows the measurement of Stokes parameters without moving the optics and secondly it results in substantial reduction of the $1 / f$ noise, now confined to frequency bands determined by the modulation period. Three methods have been proposed for phase modulation in millimetre cosmology instruments. The first is a rotating half-wave plate in front of the array that modulates the polarization continuously at a rate equal to four times the plate rotation frequency. This is relatively simple to implement since it only requires a single rotating plate for the whole array. However, the passage of the wave through a retardation

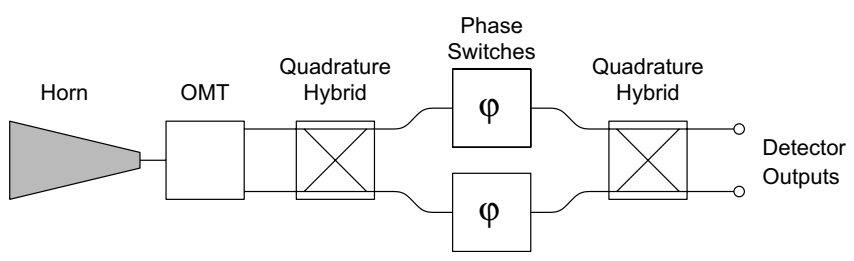

Fig. 1. The layout of a pseudo-correlation polarimeter. We propose that the hybrids, the phase switches and the detectors be fabricated in planar circuit assemblies. plate could impart polarisation systematics as a result of tiny anisotropies in the plate material. Also, the plate itself constitutes a scattering aperture that could easily degrade the optical performance of the telescope. Another method that has been considered for the clover project is to rotate a waveguide section in the individual polarisation channels. This method however can only be effective at the lower end of the millimetre spectrum and requires the employment of a large number of tiny motors in a cryogenic environment, which is by no means straight-forward. Finally, Faraday rotator ferrite rods, mounted in cylindrical waveguides have been investigated for phase modulation by the BICEP team [9], but as far as we know, they found them very difficult to mass produce and to suffer from significant RF losses.

Our conclusion therefore is that there is an urgent need for the development of phase modulation using planar circuits [10] for the present and next generation of cosmology instruments. Success in this program will have huge advantages. The planar circuits will be relatively easy to fabricate at high frequencies and will not use rotating components or obstacles that introduce systematics. More importantly, it will allow the fabrication of the whole polarimeter in planar circuit technology with the horn and OMT only implemented in waveguide, while the rest of the array will be integrated in the detector block. This will not only solve the problem of phase modulation at high frequencies, but will pave the way for fabricating huge arrays reliably and cheaply.

The phase switch circuit consists of two main components, the millimetre on/off switch and the phase shift circuit. In a previous publication [10], we have discussed ways of realising the millimetre switch. In this paper we will present experimental results from fabricated devices and a design that integrates the switch into a microstrip phase shift circuit, yielding a complete millimetre-wave phase switch system in superconducting microstrip.

\section{The superconducting nanostrip switch}

\subsection{Properties of the $R F$ circuit}

Consider an electromagnetic wave propagating along a lossless and matched planar transmission line. A suitable millimetre switch allows low loss transmission of the wave in the open mode and causes high reflection in the closed mode. This operation may be achieved by inserting a carefully selected load of impedance $Z$ across the terminals of a transmission line of characteristic impedance $Z_{0}$. If the impedance of the load is made to change between $Z_{\text {on }} \ll Z_{0}$, and $Z_{\text {off }} \gg Z_{0}$, the device switches from the closed to the open state, respectively.

A superconducting nanostrip can be realised by depositing a capacitively coupled thin superconducting strip across the electrodes of the transmission line. A schematic view and a lumped element model of the switch are shown in Figs. 2a and b, respectively. By applying a suitable bias 


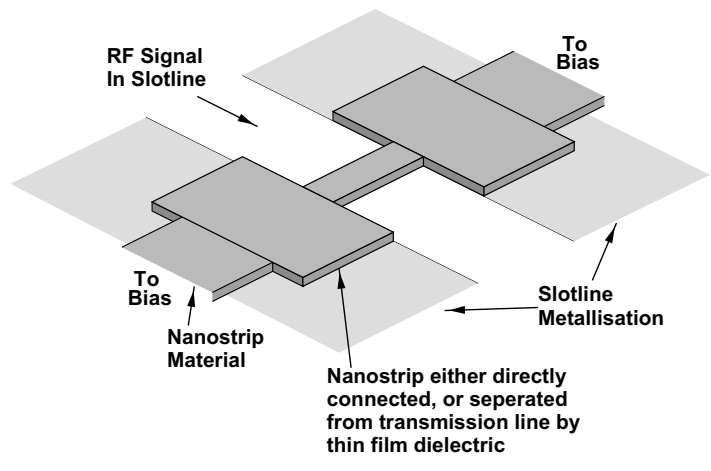

(a) Nanostrip geometry

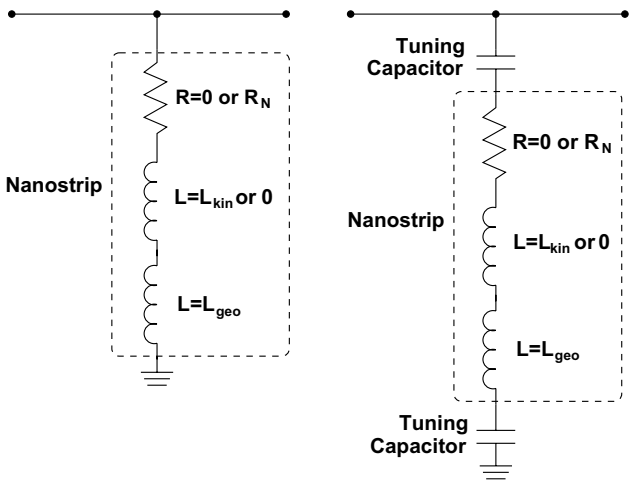

(b) Equivalent circuits for the simple and capacitively coupled "nanostrips

Fig. 2. (a) Geometry of a nanostrip switch in slotline transmission line. (b) Equivalent circuits used to model both the directly and capacitively coupled nanostrip switches. In the superconducting state the nanostrip shows zero resistance and a small kinetic inductance $\left(L_{\mathrm{kin}}\right)$, while in the normal metal state it shows a high resistance $\left(R_{\mathrm{N}}\right)$ and no kinetic inductance. An inductance $\left(L_{\mathrm{geo}}\right)$ due to the geometry of the nanostrip is present in both states.

voltage signal, across the terminals of a nanostrip which is cooled below its transition temperature, it is made to switch from the superconducting state with an impedance given by

$Z_{\text {on }}=\mathrm{i} \omega\left(L_{\mathrm{g}}+L_{\mathrm{k}}\right)+2 / \mathrm{i} \omega C=\left(2-\omega^{2} L C\right) / \mathrm{i} \omega C$

to the normal state with an impedance

$Z_{\text {off }}=R_{\mathrm{N}}+\mathrm{i} \omega L_{\mathrm{g}}+2 / \mathrm{i} \omega C=R_{\mathrm{N}}+\left(2-\omega^{2} L_{\mathrm{g}} C\right) / \mathrm{i} \omega C$,

where $R_{\mathrm{N}}$ is the normal resistance and $L_{\mathrm{g}}, L_{\mathrm{k}}$ and $L$ are, respectively the geometrical, kinetic and total inductances of the strip. At resonance $\left(\omega^{2} L C=2\right)$, the impedance in the superconducting state can be made very small causing perfect reflection (switch closed). The impedance in the normal state is dominated by the normal resistance of the nanostrip providing $\omega L \ll R_{\mathrm{N}}$, which can easily be satisfied at millimetre wavelengths. The material and geometry of the nanostrip are therefore chosen to make $R_{\mathrm{N}}$ as high as possible (switch open).
Consider for example a niobium nitride thin film nanostrip of length $l=5 \mu \mathrm{m}$ deposited across the terminals of a $50 \Omega$ transmission line. For a $1 \mu \mathrm{m}$ wide and $20 \mathrm{~nm}$ thick nanostrip, the normal resistance (the resistivity of $\mathrm{NbN}$ is $200 \mu \Omega \mathrm{cm}$ ) is $\approx 500 \Omega$ and the geometrical inductance is $\approx 3 \mathrm{pH}$. Assuming a London penetration depth of $200 \mathrm{~nm}$, the kinetic inductance at frequencies well below the superconducting gap is $12.6 \mathrm{pH}$. The scattering parameters of the capacitively coupled nanostrip using the above values are plotted in Fig. 3, where a capacitance value of $65 \mathrm{fF}$ was chosen to give a centre frequency of $225 \mathrm{GHz}$.

The fractional bandwidth of the LRC circuit is given by $\frac{\Delta \omega}{\omega}=\frac{Z_{0}}{\omega L}$.

From Fig. 3 we can see that the $-15 \mathrm{~dB}$ bandwidth of the switch is $50 \mathrm{GHz}$ and the $-13 \mathrm{~dB}$ is $70 \mathrm{GHz}$. This fractional band is sufficient for many applications including the $\mathrm{CMB}$ phase modulation. Increasing the bandwidth

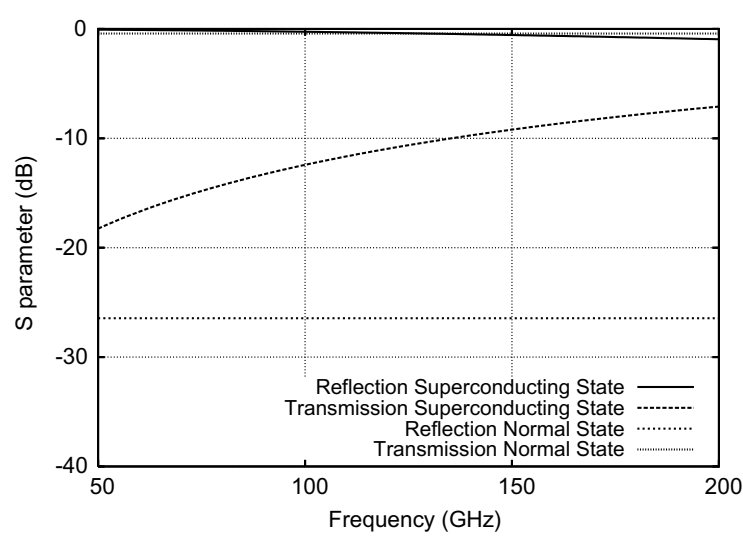

(a) Directly coupled nanostrip

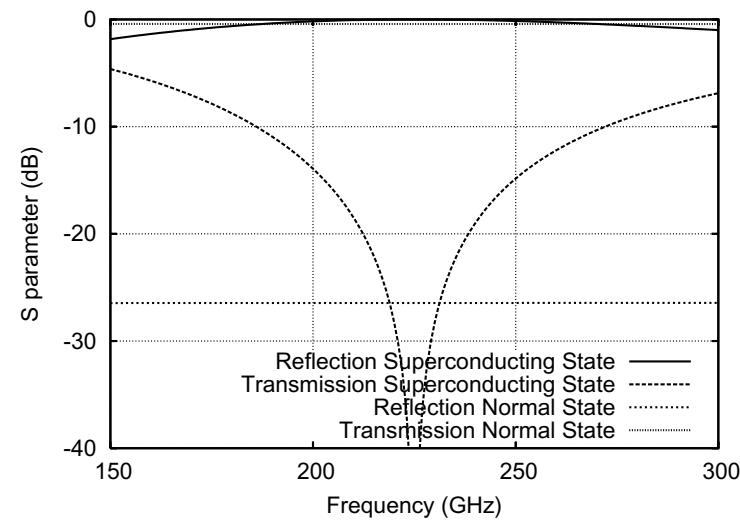

(b) Capacitively coupled nanostrip

Fig. 3. Reflection and transmission by nanostrip shunt switches. (a) the nanostrip is directly coupled to the transmission line and (b) the nanostrip is coupled to the transmission line via $65 \mathrm{fF}$ thin film capacitors, giving a centre frequency of $225 \mathrm{GHz}$. In both cases the transmission line impedance is $50 \Omega$ and the nanostrip is a $5 \mu \mathrm{m}$ long, $1.0 \mu \mathrm{m}$ wide and $20 \mathrm{~nm}$ thick niobium nitride thin film with a critical current density of $510^{3} \mathrm{~A} \mu \mathrm{m}^{-2}$, London penetration depth $200 \mathrm{~nm}$ and a normal state resistivity of $2.0 \Omega \mu \mathrm{m}$. 
significantly requires decreasing $L$, while still maintaining the condition of $R_{\mathrm{N}}>Z_{0}$. This could be achieved by either increasing the resistivity of the material, so that the geometrical inductance can be reduced, while keeping high normal resistance or by reducing the penetration depth of the superconductor. It is worthwhile mentioning that nanostrip switching can be obtained directly, without capacitive coupling, as can be seen from Fig. 3. However, the performance above $200 \mathrm{GHz}$ is limited, hence we will only use it for preliminary testing of the devices.

\subsection{Physical properties of the nanostrip}

A low RF resistance of the nanostrip to radiation in the superconducting state, can only be obtained if the energy gap is higher than the photon energy, i.e.

$h v<2 \Delta=k T_{\mathrm{c}}$.

To satisfy this condition in the highest frequency band of interest to the CMB observations (centred at approximately $300 \mathrm{GHz}$ ), the transition temperature of the material must be chosen so that $T_{\mathrm{c}}>\sim 5 \mathrm{~K}$. Only a few metallic materials with such high transition temperature are suitable for this purpose, with Niobium $\left(T_{\mathrm{c}}=9.2 \mathrm{~K}\right)$ and niobium nitride $\left(T_{\mathrm{c}}=9-14 \mathrm{~K}\right)$ being the most commonly employed in very thin film deposition.

A high performance nanostrip switch exhibits a large change in impedance between the normal and the superconducting states. This requires the normal resistance of the microstrip $R_{\mathrm{N}}=\rho \frac{l}{w t}$ where $l$ is the width $w$ is the width, $t$ is the thickness and $\rho$ is the resistivity, to be high relative to the kinetic inductance. However, a thin superconducting strip exhibits parasitic inductance, due to two effects: the self inductance which is determined by the geometry of the strip, with

$L_{\text {geo }} \approx 0.2 l\left(\frac{1}{2}+\ln \left(\frac{2 l}{w+t}\right)+0.11\left(\frac{w+t}{l}\right)\right) \mu H$.

and the kinetic inductance of the nanostrip in the superconducting state by

$L_{\text {kin }}^{\mathrm{SC}}=\mu_{0} \frac{l \lambda}{w} \operatorname{coth} \frac{t}{\lambda} \sim \mu_{0} \lambda^{2} \frac{l}{w t}$,

where $\lambda$ is the London penetration depth of the material (the kinetic inductance in the normal state is insignificant). As can be seen, the ratio of the kinetic inductance in the superconducting state to the resistance in the normal state is almost independent of the geometry of the nanostrip, and is mainly determined by the choice of material. Assuming that $\omega L_{\mathrm{g}} \ll R_{\mathrm{N}}$ (which can easily be satisfied for the values described in Fig. 3) then for direct coupling $(C=\infty)$ Eqs. (3) and (4) yield.

$\frac{Z_{\text {off }}}{Z_{\text {on }}} \approx \frac{\rho}{\mu_{0} \omega \lambda^{2}}$,

which is independent on the strip geometry. Fig. 6 illustrates that a niobium nitride nanostrip can indeed make a good millimetre switch for the proposed dimensions, hence the choice of the nanostrip geometry is also made to guarantee that $R_{\mathrm{N}} \gg Z_{0}$, small geometrical inductance and easy fabrication of the device. For a $50 \Omega$ transmission line, a $20 \mathrm{~nm}$ thick, $1.0 \mu \mathrm{m}$ wide niobium nitride thin film gives a normal state resistance of $500 \Omega$, for a convenient length of $5 \mu \mathrm{m}$, which is the typical gap in a $50 \Omega$ unilateral finline. The geometric inductance of this nanostrip is 2.8 $\mathrm{pH}$ which satisfies the requirements of Eq. (8).

We shall now consider the thermal properties of the nanostrip. A good phase switch should not dissipate a large amount of power, in particular when it is used in a space instrument or in a very large array. In the case of voltage biasing, when the bias is switched from zero to a finite value, the current through the device rises with a slope determined by the nanostrip inductance, until it reaches the critical superconducting current. The nanostrip then switches to the normal state, and dissipates electrical power according to $P=V^{2} / R_{\mathrm{N}}$. This input electrical power will be balanced by the dissipation of heat via the electron-phonon interaction (which is the dominant thermal conduction mechanism in thin superconducting films). The power dissipation in an electron-phonon interaction may be written as

$P=\alpha\left(T_{\mathrm{e}}\right) w l t\left(T_{\mathrm{e}}^{n}-T_{1}^{n}\right)$,

where $\alpha$ is the coefficient of the electron-phonon interaction, wlt is the volume of the nanostrip, $T_{1}$ is the lattice temperature and $T_{\mathrm{e}}$ is the electron temperature of the electron gas, approximately equal to $T_{\mathrm{c}}$ for a nanostrip biased at the superconducting transition. For a $\mathrm{NbN}$ strip at $\sim 5 \mathrm{~K}$ it is found that the coefficient $\alpha$ is given by [11]

$\alpha(T)=\frac{C_{e}}{n T^{(n-1)} \tau_{e p h}}$,

where $n=3.6, \tau_{\text {eph }}$ is the electron-phonon interaction time and $C_{\mathrm{e}}$ is the electron heat capacity. A typical niobium nitride nanostrip at $T_{\mathrm{e}}=9 \mathrm{~K}$ will therefore have a value of $\alpha \sim 7 \times 10^{-8} \mathrm{~W} /(\mu \mathrm{m})^{3} \mathrm{~K}^{-3.6}[12]$ and assuming dimensions of $l=5 \mu \mathrm{m}, w=0.3 \mu \mathrm{m}$ and $t=20 \mathrm{~nm}$, we obtain a power dissipation value of $\sim 5 \mu \mathrm{W}$ to keep the nanostrip in the normal state. It may be possible to lower this figure by reducing the volume of the nanostrip or isolating the strip on a small $\mathrm{SiN}$ island to lower the value of the conductance.

In order to switch the nanostrip from the superconducting state to the normal state, we have to supply enough current to exceed the critical superconducting current of the nanostrip. Assuming that the critical current will be around $100 \mu \mathrm{A}$ and the normal resistance $1 \mathrm{k} \Omega$ then On switching, a transient power of around $10 \mu \mathrm{W}$ will be dissipated in the now resistive nanostrip, which is similar to the value obtained in the previous calculation.

\subsection{Fabrication of nanostrip switches}

The test devices were fabricated at Chalmers MC2 by depositing the nanostrip across the terminals of a back-to 
back unilateral finline on a $200 \mu \mathrm{m}$ thick quartz substrate. The fabrication process of the finline coupled nanostrip devices includes two lithography steps, namely a photo lithography for fabricating the contact pads and e-beam lithography for depositing the $\mathrm{NbN}$ nanostructures. The whole fabrication procedure is shown in Fig. 4.

First, the $\mathrm{NbN}$ film is deposited on a quartz substrate by magnetron sputtering of a niobium cathode in argon-nitrogen mixture plasma (Fig. 4a). The $\mathrm{NbN}$ molecules are deposited on a hot substrate which is heated by a lamp before and during the deposition process. Two layers of resist are then spun on the wafer: a lift-off layer LOL and a photoresist Shipley S-1813. After baking of both resist layers, the wafer is exposed to UV radiation at wavelength of $400 \mathrm{~nm}$ through a photo mask to pattern the gold pads and finline. After development of the photoresist, an undercut is formed in the lift-off layer (Fig. 4b). The wafer is then cleaned in the etching process by a soft oxygen plasma and a thin layer of chromium and $500 \AA$ of gold are evaporated through the resist mask and lifted off (Fig. 4c).

The next process step is the e-beam lithography for patterning the $\mathrm{NbN}$ nanostructures. First, the negative e-beam resist SAL-601 is spun on the wafer and baked (Fig. 4d). Then the $\mathrm{NbN}$ structures are patterned by two e-beam exposures, the coarse one at the 4th lens for larger structures and then at the 5th lens for fine patterning of nano- structures. After the development of the e-beam resist, the $\mathrm{NbN}$ structures are patterned by the reactive ion etching in CF4 plasma (Fig. 4e). The rest of the resist is then removed in Shipley 1165 remover (Fig. 4f) and finally the wafer is cut using a diamond saw and cleaned in oxygen plasma etching process.

An example device is shown in Fig. 5 in conjunction with a $5 \mu \mathrm{m}$ long nanostrip. The AFM image shows clearly that the nanostrip has neatly been fabricated. The unilateral finline transmission line was chosen for these initial tests, as a result of the simplicity in fabricating a coplanar structure and because it offers straight-forward coupling of $\mathrm{RF}$ power to the device from waveguide, and from the device to the detector in a single split-block (see Section 5).

The finline taper was synthesised using the spectral domain analysis numerical method which was verified aginst HFSS simulations. For RF testing the device will be mounted in the E-plane of an WR-4 waveguide. The serrations act as $\lambda / 4$ open-ended stubs to prevent the propagation in the waveguide groove that supports the chip. The DC and RF testing will be discussed in later sections.

\section{The millimetre-wave phase switch}

There are several RF circuits that allow the phase of a signal to be switched between two values. The conceptually

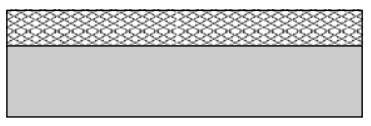

$\mathrm{NbN}$ Film

Substrate

(a)

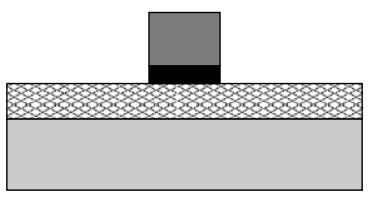

Au 500 A

Cr 100 A

$\mathrm{NbN}$ Film

Substrate

(c)

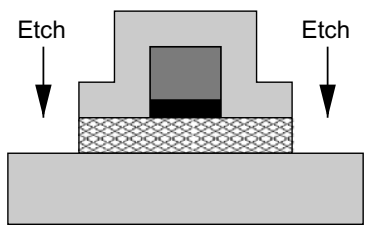

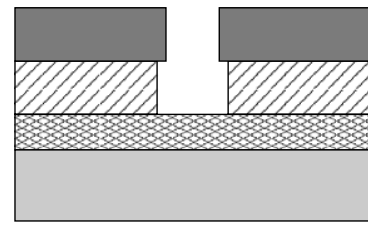

Photoresist

LOL

NbN Film

Substrate

(b)
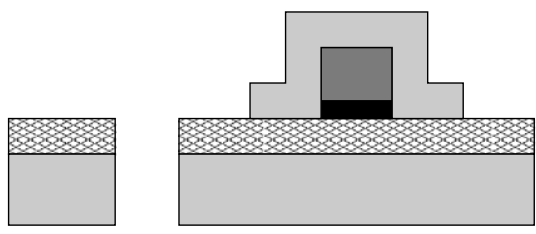

Negative

E-beam

Resist

NbN Film

Substrate

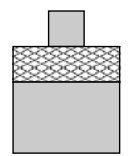

(d)

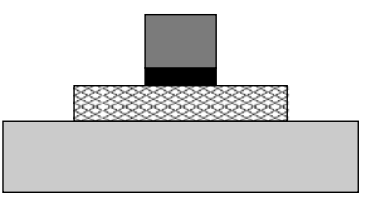

$\mathrm{Cr}$

$\mathrm{NbN}$ Film

Substrate

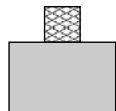

(e)

Fig. 4. Fabrication of the nanostrip across a unilateral finline. In each step, the figure on the left describes fabrication of the finline electrodes, while the smaller figure on the right describes the fabrication of the nanostrip. 


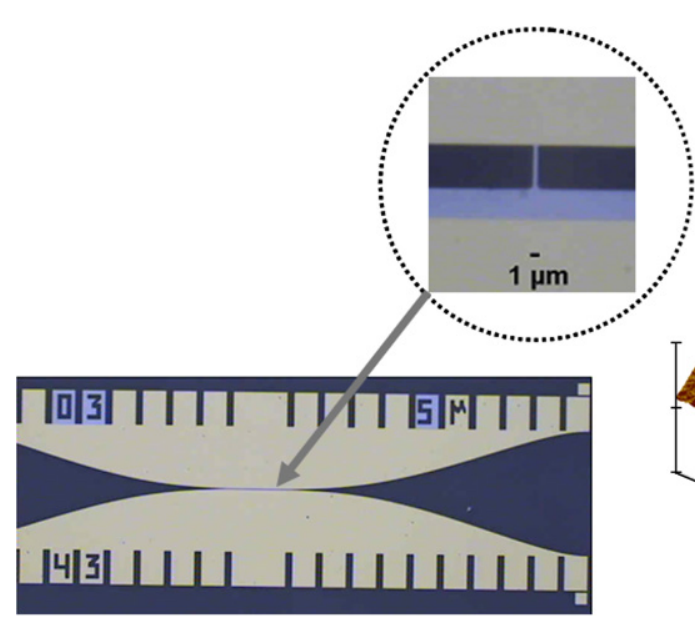

(a)

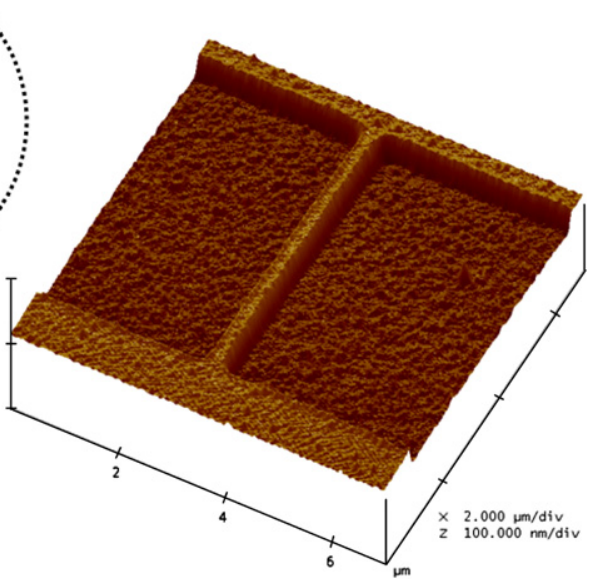

(b)

Fig. 5. (a) An optical image of the $\mathrm{NbN}$ nanostrip switch showing the back-to back finline device. (b) An AFM image of the nanostrip. The $x$-axis scale is $2 \mu \mathrm{m} / \mathrm{div}$ and the $z$-axis is $100 \mathrm{~nm} / \mathrm{div}$.

simplest phase switch circuit consists of two delay line arms of length difference $l$, one of which is isolated, while the other is connected. The switch makes the signal pass through one arm or the other with a phase difference at the output given by $\Delta \phi=\beta l$, where $\beta$ is the propagation constant in the delay lines.

An interesting realisation of the delay line design is the Schiffman phase switch delay line [13] which exhibits a small phase error, over a broad band, between switch states by balancing the dispersion in two different section of coupled lines. This gives a phase switch with a very small phase error over a large bandwidth . However, balancing the dispersion to give this small phase error requires about $-6 \mathrm{~dB}$ of coupling in one of the arms, which is very difficult to achieve in the superconducting transmission line technology we have been considering for these devices.

An alternative design is the quadrature coupled phase shifter [14] shown in Fig. 6. This circuit consists of a quad- rature hybrid with the output ports connected to shunt switched open stubs. With both switches in the closed state, signals from the input are split equally between the hybrid outputs, where they reflect from the switches before recombining in phase at the isolated input to the hybrid. With both switches in the open state, the reflected signals at the hybrid outputs experience a phase delay equal to twice the length of the stubs when being reflected. This allows an arbitrary phase shift, determined by the length of the stubs, to be generated.

Notice that the quadrature coupler circuits requires only two switches per phase bit, and only one control signal is needed, as both switches are in the same state at all times. The bandwidth of this design can be significantly improved by adding extra loops of transmission line to the hybrid as shown in Fig. 6b.

A schematic diagram showing the proposed realisation of the millimetre phase switch circuit is shown in Fig. 7.

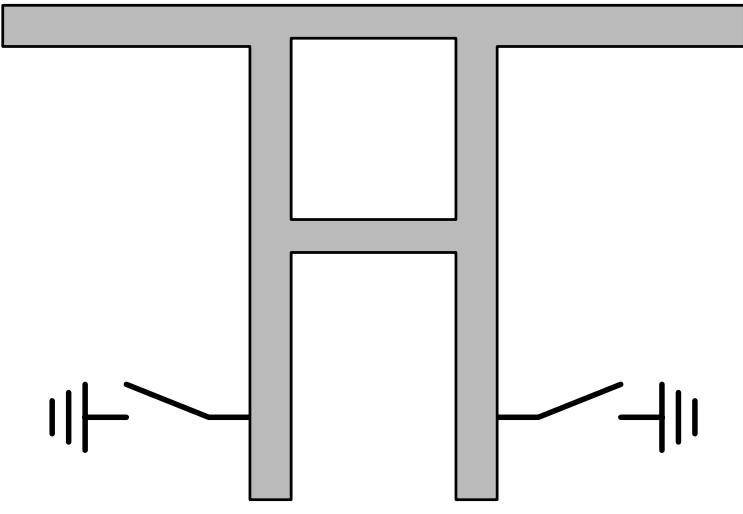

(a) Quadrature phase shifter

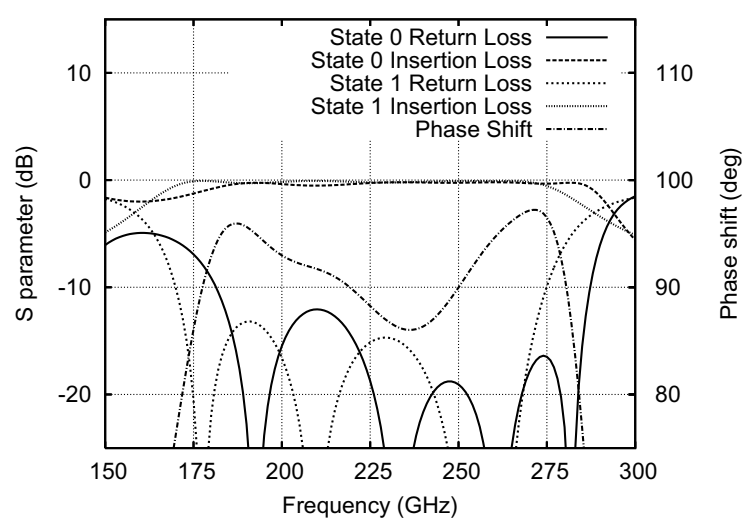

(b)

Fig. 6. (a) Quadrature coupler phase switch circuit. The square transmission line structure is a quadrature hybrid, with the input and isolated ports at the top, and the output ports at the base. Shunt switches are position one quarter wavelength from the outputs, and switch one eighth wavelength open stubs. (b) Performance of this phase shift circuit using a two sections quadrature hybrid. 


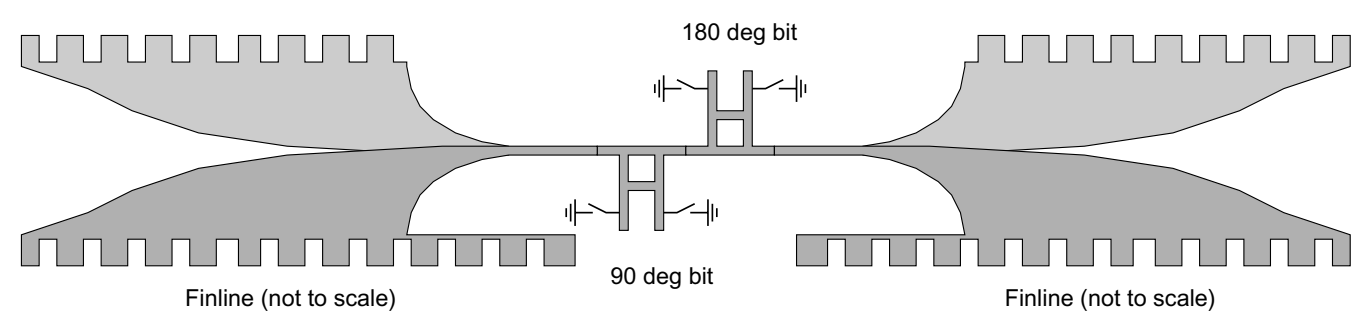

Fig. 7. A schematic millimetre phase switch circuit. Notice that the back-to-back structure was only chosen for testing but in a real design one port is likely to end in microstrip.

The input RF signal is fed to the circuit from the waveguide via an antipodal finline taper. These tapers have already been employed in conjunction with high frequency superconducting circuits and have shown excellent performance at frequencies as high as $700 \mathrm{GHz}$. The phase shift circuit is a quadrature coupler with the input and isolation ports at the top and outputs at the bottom. Two independent switches are used in order to switch the phase by both 90 and $180^{\circ}$ as required by the pseudo-correlator (see Section 1). The back-to-back structure is only presented for possible Vector Network Analyser (VNA) tests, but in practice, the outputs of the phase switch are coupled to, hybrids or other planar components leading to the detectors; all realised in microstrip circuits.

In Fig. 8 we illustrate the method of fabricating the nanostrip across a microstrip transmission line. The nanostrip is first deposited on the quartz substrate with contact pads as shown above. The microstrip is then deposited on the substrate leaving an area of approximately $10 \mu \mathrm{m}^{2}$ uncovered. The dielectric layer oxide $(400 \mathrm{~nm}$ of $\mathrm{SiO})$ is then deposited, again leaving the gap in the ground plane uncovered. Finally the microstrip is deposited, so that the part crossing the gap is connected to the nanostrip via one of the contact pads. Particular care must be taken to ensure that step coverage problems do no occur where the microstrip transmission line crosses the edges of the gap in the ground plane and dielectric layer.

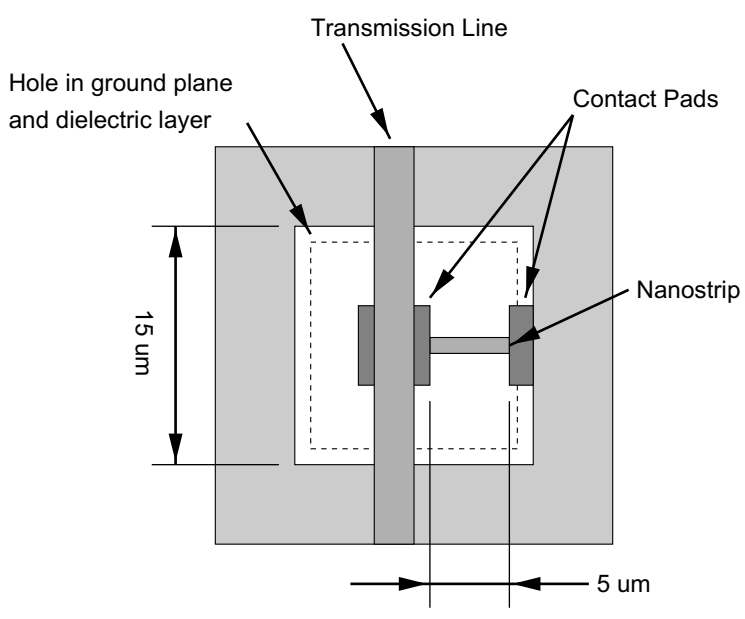

Fig. 8. Integration of a nanostrip in a microstrip transmission line. The nanostrip is deposited on the substrate across a gap in the ground plane.
We have simulated the RF behaviour of the microstrip nanostrip switch and shown that it is similar to the results obtained from the lumped element model. In other words, the existence of the gap in the ground plane does not significantly degrade the behaviour of the microstrip transmission line.

The designs given in this Figs. 7 and 8 constitute a complete scheme for fabricating a planar $90^{\circ}$ and $180^{\circ}$ phase switch. The processing requires only three masks over those required for the nanostrip fabrication; one to deposit the ground plane and the bottom layer of the finline, a second to deposit the $\mathrm{SiO}$ layer for the microstrip, and a third to deposit the microstrip circuits and the finline upper layer.

\section{Preliminary DC tests}

Several samples of the finline nanostrip devices were selected for DC tests at $4.2 \mathrm{~K}$. The finline gap for these devices was $5 \mu \mathrm{m}$ corresponding to a characteristic impedance of $69 \Omega$. The thickness of $\mathrm{NbN}$ nanostrip film was $22 \mathrm{~nm}$ and width ranged from 0.4 to $2 \mu \mathrm{m}$.

The measurements have shown that the critical temperature of the $\mathrm{NbN}$ film is about $9 \mathrm{~K}$, and the critical currents for different nanostrip widths varies from 12 to $50 \mu \mathrm{A}$, which corresponds to a critical current density of $150 \mathrm{kA} /$ $\mu \mathrm{m}^{2}$. An example of a typical measured IV curve of the nanostrip, using a two wire configuration, is shown in Fig. 9a. For a strip width of $0.5 \mu \mathrm{m}$ and thickness of $22 \mathrm{~nm}$, the measured value of the critical current was $38 \mu \mathrm{A}$.

The time-domain measurements of the switching was performed at frequencies from $100 \mathrm{~Hz}$ to $100 \mathrm{kHz}$. Notice that the bias voltage curve (upper line) in Fig. $9 b$ has an offset value and yet the measured voltage across the nanostrip in the superconducting state was zero (bottom blue trace). This illustrates that the nanostrip has indeed shorted the signal in the superconducting state. A time delay of approximately $0.5 \mu \mathrm{s}$, was observed at the leading edges of the modulation voltage, which is negligible compared to switching period as shown in Fig. 9b. Moreover, the observed delay is in fact caused by the RC-time constant of the measurement setup rather than by the switching properties of the $\mathrm{NbN}$ nanostrip. This was confirmed experimentally by measuring the RC-delay of the experimental setup without the sample. This result is also consistent with previously published data which reported that the 


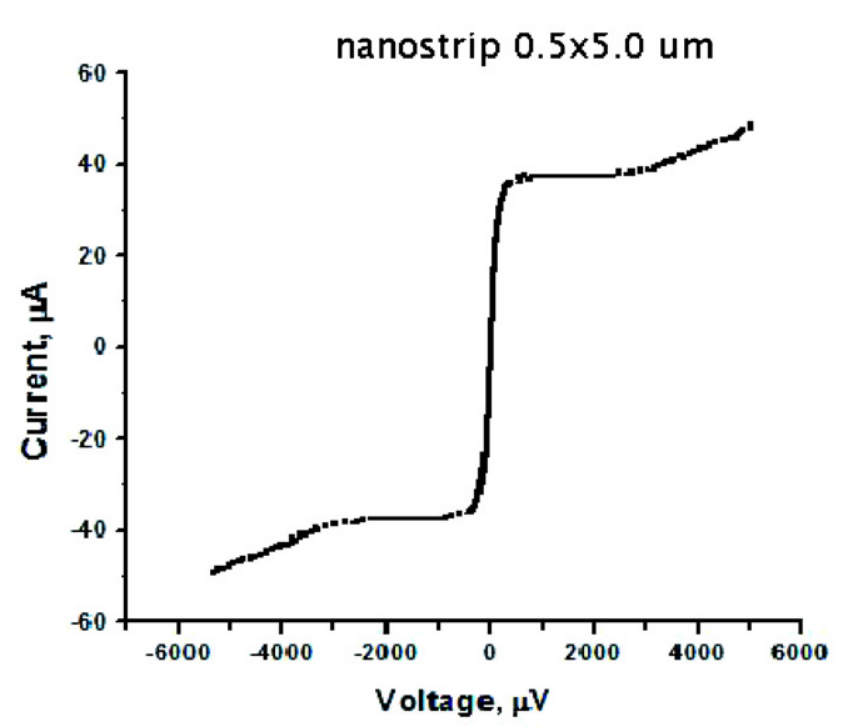

(a) IV curve

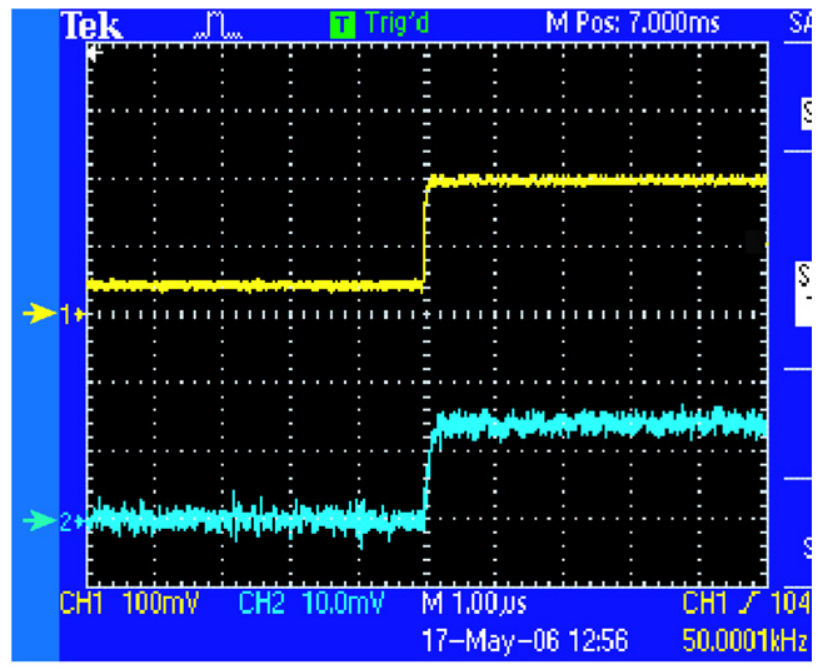

(b) DC test measurement

Fig. 9. (a) $I-V$ curve of a finline nanostrip measured at $4.2 \mathrm{~K}$. The length of the strip is $5 \mu \mathrm{m}$, the width is $0.5 \mu \mathrm{m}$, the thickness is $22 \mathrm{~nm}$ and the current density is $38 \mu \mathrm{A}$. (b) DC test of the finline nanostrip. The upper trace is the bias current and the lower is the output voltage across the strip. The arrows indicate the zero signal level.

delay in switching of a superconductive nanostrip should not exceed $30 \mathrm{ps}$ [15]. The switching speed of the nanostrip is however well above what is required for large format interferometers which is of the order of $100 \mathrm{kHz}$.

The above measurements are extremely encouraging and confirm that a nanostrip across a transmission line is an excellent DC switch. RF testing of the switch is in progress and can be easily performed by mounting the device in a split-block using the same techniques employed in conjunction of SIS mixers. The device is inserted in a waveguide groove followed by another finline device which contain as SIS tunnel junction which is used as a direct detector. The response of the switch to RF signal can then be assessed by measuring the tunnelling current across the SIS junction. A split-block design similar to the one that will be employed in our testing of the phase switch has previously been reported [16]. An alternative more elegant method for testing the RF behaviour is to fabricate the detector (which can be an SIS or an SIN tunnel junction) across the same device of the nanostrip.

\section{Conclusions}

We have presented novel designs for a millimetre switch based on a superconducting nanostrip across a transmission line with capacitive coupling. We have fabricated several samples using a $5 \mu \mathrm{m}$ long nanostrip across the gap of a unilateral finline. Our DC tests of the finline switch have shown that the response time is sufficiently fast for phase modulation in astronomical instrument. In fact the determination of the delay time of the switch was limited by the time constant of the measurement system $(5 \mu \mathrm{s})$.

Next we investigated planar phase shift circuits and presented designs that are simple to fabricate in superconduc- ting microstrip. The bandwidth of these circuit can be made sufficiently large by using successive hybrid sections. Such circuits are commonly used in microwave circuits and extending the design to millimetre wavelengths is straightforward.

Finally we presented a superconducting nanostrip switch integrated in a microstrip phase shift circuit. The $\mathrm{RF}$ signal which is usually received by a horn, is fed to the microstrip circuit using an antipodal finline taper. We have shown how this structure can easily be fabricated and RF tested. To the best of our knowledge this is the fist time a fully planar passive circuits design of a millimetre phase switch has been reported. The planar phase switch will have several advantages over existing designs:

- The losses are much smaller since superconducting structures are used. The fast response of the circuit allows a wide range of modulation frequencies. The main limitation on the performance comes from the finite geometrical inductance of the nanostrip, but from Fig. 3 we can see that if capacitive coupling is used then the transmission loss can be made very small.

- The RF bandwidth is limited by the bandwidth of the switch LRC circuit and of the hybrid of the phase circuit. The simulations presented above show that the useful bandwidth is sufficient for the requirements of cosmology instruments which is less than $30 \%$.

- It can be fabricated reliably and mass produced relatively cheaply and rapidly. This is because no mechanical components are required as the phase switch can be integrated into the detector block.

Finally, the proposed design allows the fabrication of the whole polarimeter, excluding the horn, in planar struc- 
tures allowing cosmology instruments to be fabricated with many thousands of detectors and reach unprecedented sensitivities.

\section{References}

[1] W. Hu, M. White, New Astronomy 2 (1997) 323.

[2] A.C.S. Readhead, S.T. Myers, T.J. Pearson, J.L. Sievers, B.S. Mason, C.R. Contaldi, J.R. Bond, R. Bustos, P. Altamirano, C. Achermann, L. Bronfmoan, J.E. Carlstrom, J.K. Cartwright, S. Casassus, C. Dickinson, W.L. Holzapfel, J.M. Kovac, E.M. Leitch, J. May, S. Padin, D. Pogosyan, M. Pospleszalski, C. Pryke, R. Reeves, M.C. Shephard, S. Torres, Science 306 (5697) (2004) 836.

[3] J.M. Kovac, E.M. Leitch, C. Pryke, J.E. Carlstrom, N.W. Halverson, W.L. Holzapfel, Nature 420 (2002) 772.

[4] L. Page, G. Hinshaw, E. Komatsu, M.R. Nolta, D.N. Spergel, C.L. Bennett, C. Barnes, R. Bean, O. Dore, M. Halpern, R.S. Hill, N Jarosik, A. Kogut, M. Limon, S.S. Meyer, N. Odegard, H.V. Peiris, G.S. Tucker, L. Verde, J.L. Weiland, E. Wollack, E.L. Wright, Threeyear Wilkinson microwave anisotropy probe observations: polarization analysis, APJS 170 (2007) 335.

[5] G. Yassin, et al, CLOVER-A novel instrument for measuring the CMB B-mode polarization, in: Proceedings of the 15th International Symposium on Space THz. Tech., Proc. 1, 2004.

[6] P. Oxley et al., Proc. SPIE 5543 (2004) 320.

[7] B.G. Keating et al., Proc. SPIE 4843 (2003) 284.
[8] B. Winstein, et al, QUIET: Goals and status, in: Proceedings of the 2nd Irvine Cosmology Conference, Beckman Center, University of California, Irvine, CA., 2006.

[9] K.S. Yoon, P.A.R. Ade, D. Barkats, J.O. Battle, E.M. Bierman, J.J. Bock, J.A. Brevik, H.C. Chiang, A. Crites, C.D.D. amd, L. Duband, G.S. Griffin, E.F. Hivon, W.L. Holzapfel, V.V. Hristov, B.G. Keating, J.M. Kovac, C.L. Kuo, A.E. Lange, E.M. Leitch, P.V. Mason, H.T. Nguyen, N. Ponthieu, Y.D. Takahashi, T. Renbarger, L.C. Weintraub, D. Woolsey, Proc. SPIE 6275 (2006) 1K.

[10] P.K. Grimes, G. Yassin, L.S. Kuzmin, P.D. Mauskopf, E. Otto, M.E. Jones, C.E. North, Proc. SPIE 6275 (2006) 25.

[11] J. Kooi, J. Baselmans, M. Hajenius, J. Gao, T.M. Klapwijk, P. Dieleman, A. Baryshev, G. de Lange, J. Appl. Phys. 101 (2007) 044511.

[12] R.S. Nebosis, A.D. Semenov, Y.P. Gousev, K.F. Renk, Rigorous analysis of a superconducting hot-electron bolometer mixer. Theory and comparison with experiment, in: Proceedings of the 7th International Symposium on Space THz. Tech, University of Virginia, Charlottesville, USA, 1996.

[13] B.M. Schiffman, IRE Trans. Microwave Theor. Tech. 4 (1958) 232.

[14] D.M. Pozar, Microwave Engineering, second ed., John Wiley and Sons, Inc., 1998.

[15] K.S. Ilín, M. Lindgren, M. Currie, A.D. Semenov, G.N. Goltsman, R. Sobolewski, Appl. Phys. Lett. 76 (2000) 2752.

[16] G. Yassin, R. Padman, S. Withington, K. Jacobs, S. Wulff, Electr. Lett. 33 (1997) 498. 